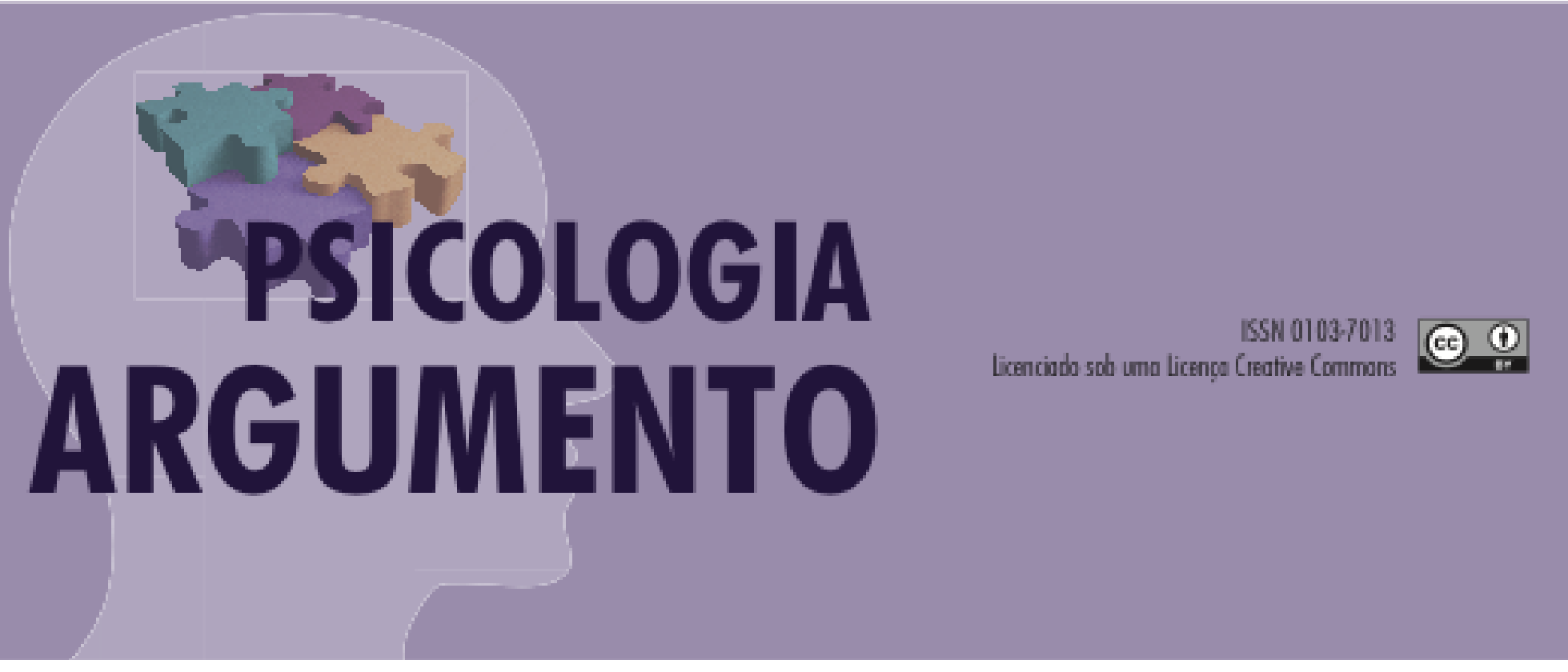

doi: http://dx.doi.org/10.7213/psicolargum.37.95.AO07

\title{
Avaliação neuropsicológica em adultos com Esclerose Múltipla: Revisão sistemática
}

Neuropsychological assessment in adults with multiple sclerosis:

Systematic review

Evaluación neuropsicológica en adultos con esclerosis múltiple:

Revisión sistemática

Mariana Kaspchak Arent

https://orcid.org/0000-0002-4440-4483

Discente do programa de mestrado Mestrado em Psicologia, da Universidade Federal do Paraná.

Curitiba, Paraná, Brasil. E-mail: marianaartigos@gmail.com

Ana Paula Almeida de Pereira

https://orcid.org/0000-0002-3779-6976

Docente do programa de Mestrado em Psicologia, da Universidade Federal do Paraná. Curitiba,

Paraná, Brasil. E-mail: anapaula_depereira@yahoo.com

Henry Koiti Sato

https://orcid.org/0000-0002-5582-1792

Coordenador do Ambulatório de Doenças Desmielinizantes do Instituto de Neurologia de

Curitiba (INC), Curitiba, Paraná, Brasil. E-mail: satohenry@gmail.com

Clovis Gomes Junior

https://orcid.org/0000-0002-5576-8143

Discente do curso de graduação em Psicologia da Universidade Federal do Paraná. Curitiba,

Paraná, Brasil.E-mail: psiclovis@gmail.com 


\begin{abstract}
Resumo
A Esclerose múltipla (EM) é considerada uma doença crônica que acomete mais frequentemente jovens adultos. Estima-se que 2,3 milhões de pessoas no mundo são afetadas pela doença. Pessoas com EM costumam apresentar alterações cognitivas e na sua qualidade de vida. A presente pesquisa visa a identificar e discutir os instrumentos de avaliação neuropsicológica utilizados atualmente com pessoas com diagnóstico de EM. Para tanto, foi realizada uma revisão sistemática da literatura. Este estudo mostrou a importância da investigação das funções cognitivas em pessoas com diagnóstico de EM. As baterias breves mais utilizadas evidenciadas na revisão sistemática são Brief International Cognitive Assessment of Multiple Sclerosis (BICAMS), Multiple Sclerosis Functional Composite Measure (MSFC) e Brief Repeatable Battery of Neuropsychological Test (BNB-N). Os testes mais utilizados na avaliação neuropsicológica de paciente com EM são PASAT e SDMT, que enfocam a avaliação da velocidade de processamento e memória operacional.
\end{abstract}

Palavras chaves: Esclerose Múltipla, avaliação neuropsicológica, testes, revisão sistemática.

\begin{abstract}
Multiple sclerosis (MS) is considered a chronic disease that more often affects young adults. It isestimated that 2.3 million people live with MS worldwide. People with MS usually have changes in their cognition and quality of life. The present research aimed to identify and discuss the instruments of neuropsychological assessment currently used with people diagnosed with MS. A systematic review of literature was carried out. This study showed the importance of investigating cognitive functions in people diagnosed with MS. The most frequently used brief batteries found were Brief International Cognitive Assessment of Multiple Sclerosis (BICAMS), Multiple Sclerosis Functional Composite Measure (MSFC) and Brief Repeatable Battery of Neuropsychological Test $(B N B-N)$. The most frequently used tests as part of the neuropsychological assessment of MS patients were PASAT and SDMT, which focus on assessing information processing speed and working memory.
\end{abstract}

Key words: Multiple Sclerosis, Neuropsychological assessment, test, systematic review.

\title{
Resumen
}

La esclerosis múltiple (EM) se considera una enfermedad crónica que afecta con mayor frecuencia a los adultos jóvenes. Se estima que 2,3 millones de personas en todo el mundo están afectadas por la enfermedad. Las personas con EM generalmente tienen cambios cognitivos y de calidad de vida. Esta investigación tiene como objetivo identificar y discutir los instrumentos de evaluación neuropsicológica que se usan actualmente con personas diagnosticadas con EM. Por lo tanto, se realizó una revisión sistemática de la literatura. Este estudio mostró la importancia de investigar las funciones cognitivas en personas diagnosticadas con EM. Las baterías breves más comúnmente utilizadas que se evidencian en la revisión sistemática son la Evaluación cognitiva internacional breve de la esclerosis múltiple (BICAMS), la Medida compuesta funcional de la esclerosis múltiple (MSFC) y la Batería repetible breve de prueba neuropsicológica (BNB-N). Las pruebas más utilizadas para la evaluación neuropsicológica de pacientes con EM son PASAT y SDMT, que se centran en la evaluación de la velocidad de procesamiento y la memoria de trabajo.

Palabras clave: esclerosis múltiple, evaluación neuropsicológica, pruebas, revisión sistemática. 


\section{Introdução}

A Esclerose Múltipla (EM) é uma doença neurodegenerativa que causa danos físicos, sensoriais e psicológicos, gerando várias deficiências. Estudos também tem apontado que a EM pode interferir no funcionamento cognitivo, sendo que $65 \%$ das pessoas com diagnóstico de EM descrevem algum nível de disfunção (Patti et al., 2015).

Os comprometimentos cognitivos mais relatados são a percepção visuoespacial, memória visual, memória verbal, atenção, funções executivas, memória de trabalho, fluência e velocidade de processamento (Giazkoulidou et al., 2019). Estudos com neuroimagem revelam uma variedade de correlatos neurais para os comprometimentos cognitivos, entre eles a atrofia cerebral, a carga total de lesão e a presença de lesões corticais (Rinaldi et al., 2010; Lanz, Hahn, \& Hildebrandt, 2007; Benedict et al., 2004). Essas disfunções cognitivas geram efeitos adversos nas atividades sociais e laborais, devido a dependência física, perda de emprego, disfunção sexual e redução da qualidade de vida (Özakbaş 2015; Leavitt, Tosto, \& Riley, 2018).

Devido à frequência de prejuízos cognitivos e do impacto desses prejuízos no cotidiano, percebe-se a importância que a avaliação neuropsicológica acompanhe o exame neurológico e colabore com o processo de tomada de decisão terapêutica. Deste modo, se faz necessário a pesquisa de ferramentas utilizadas para avaliação do comprometimento cognitivo em pessoas com diagnóstico de EM, a fim de identificar e discutir a características dos instrumentos de avaliação neuropsicológica utilizados para avaliar este grupo.

\section{Método}

Trata-se de uma revisão sistemática da literatura cientifica, com objetivo de estudar a avaliação da cognição de adultos com diagnóstico de EM. Reconhece-se a importância da atualização da área e que as revisões sistemáticas podem ser usadas como base para práticas clínicas.

Foi realizada uma busca nas bases de dados BIREME, LILACS, PePSIC, PubMed e SciELO. Os seguintes descritores foram incluídos nesta pesquisa "multiple sclerosis", 
"cognition", "cognitive profile" e "neuropsychology". A pesquisa em cada base de dados foi realizada com o descritor "multiple sclerosis" unido ao conector "and" e, então, ligado às demais palavras chaves selecionadas. A revisão sistemática foi restrita ao período de 2013 a 2017, com objetivo de pesquisar artigos recentes sobre o tema.

A presente revisão sistemática utilizou como base os procedimentos do método Preferred Reporting Items for Systematic reviews and Meta-Analysis (PRISMA). O fluxo das informações passou por diferentes fases do PRISMA, iniciando pela identificação, posteriormente a seleção e elegibilidade, por fim a inclusão da publicação na amostra dos artigos revisados (Juni \& Egger, 2009).

As pesquisas selecionadas tiveram como critério de inclusão: (a) estudos empíricos publicados entre 2013-2017 nos quais a cognição foi avaliada em pessoas com diagnóstico de EM; (b) artigos publicados em periódicos científicos que apresentam a utilização de testes neuropsicológicos em seus procedimentos; (c) ausência de comorbidades neurológicas ou psiquiátricas. Os critérios de exclusão foram: (a) amostras com faixa etária abaixo de 12 anos; (b) estudo de casos. A aplicação dos critérios e a escolha dos artigos foi realizada por meio da leitura dos títulos, palavras chaves, resumos e pelo método de cada pesquisa.

Análise de dados

Primeiramente, foi elaborado um quadro com informações que caracterizavam todos os artigos incluídos na amostra (referência, objetivo, método, subtipo clínico, EDSS e conclusão). Posteriormente, as características dos periódicos nos quais os artigos foram publicados foram descritas (por exemplo ano de publicação, área de conhecimento dos periódicos).

\section{Resultados}

A busca em todas as bases de dados resultou em 144 artigos que aplicados os critérios de inclusão e exclusão, resultaram em um total de 58 artigos a serem avaliados. A base de dados PubMed foi a que mais apresentou artigos indexados, resultando num total de 44 artigos a serem avaliados, em listagem inicial de 80 artigos encontrados. A segunda base de dados com maior número de estudos foi a BIREME, com dez artigos 
selecionados num total de 42 que foram encontradas na base. A pesquisa na Lilacs retornou 14 artigos, dos quais 5 foram selecionados. A Scielo listou 6 artigos e nenhum foi selecionado. Por fim, a busca na base Pepsic não encontrou resultados para a pesquisa.

Entre os artigos excluídos, 19 eram duplicados e os demais não estavam dentro dos critérios de inclusão e exclusão pré-estabelecidos. A maior porcentagem dos artigos eliminados não utilizava na sua metodologia testagem neuropsicológica. Sendo que a maioria dos artigos excluídos com enfoque em reabilitação cognitiva utilizaram como parâmetro de melhora exclusivamente ressonância magnética funcional para análise das mudanças funcionais e estruturais do cérebro. Também foram excluídos artigos que tiveram como enfoque outras doenças autoimunes e avaliação psicológica em outros contextos que não o neuropsicológico. Dois artigos não foram encontrados na íntegra para análise. O fluxograma geral dos artigos incluídos na pesquisa encontra-se na Figura 1.

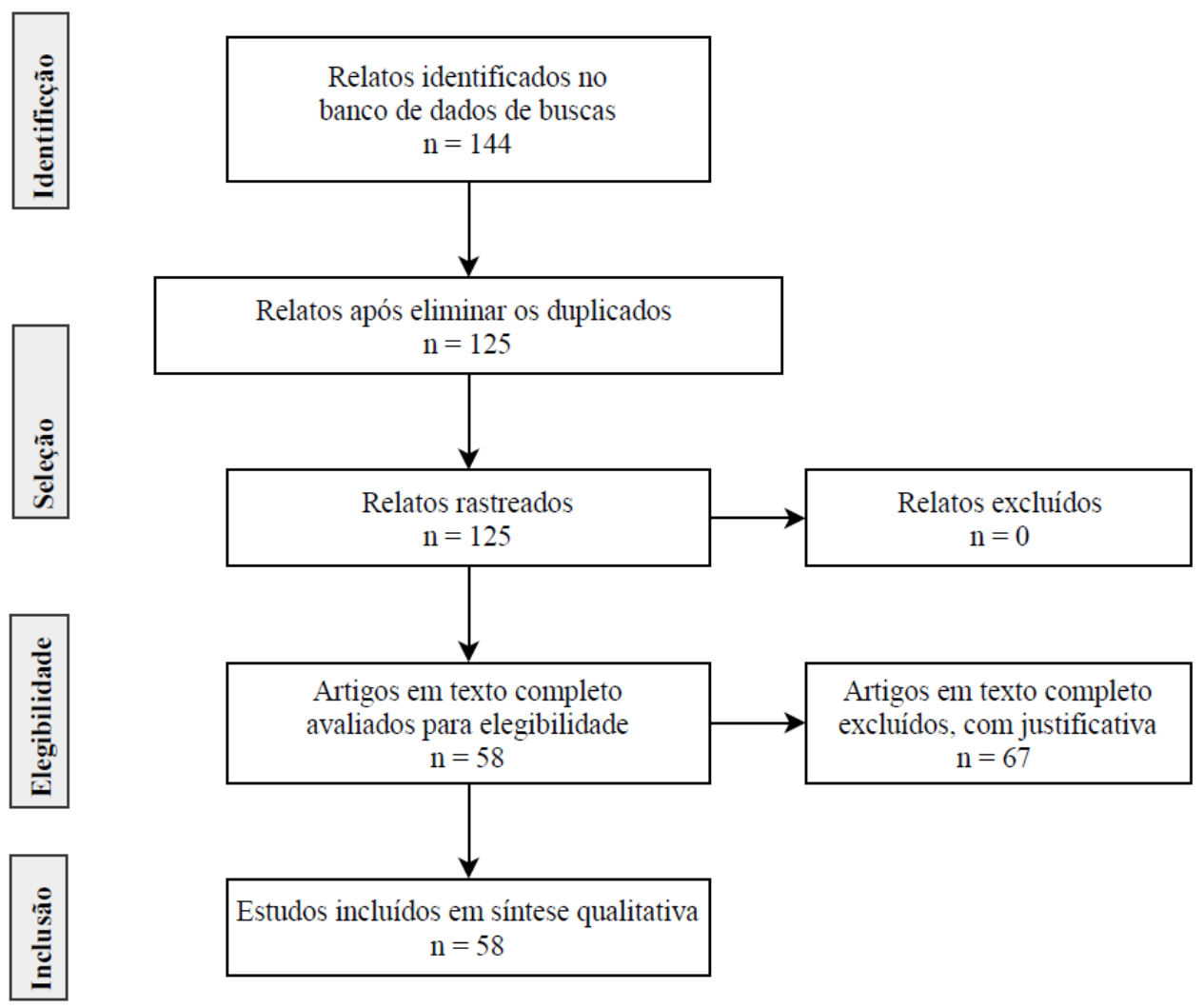

Figura 1. Processo de identificação e seleção dos artigos. 
Durante o período estudado a média de artigos publicados anualmente foi de 11,8 artigos/ano. Em 2016 obteve-se a maior quantidade de artigos publicados, somando 17 artigos, com total de $29 \%$ das publicações pesquisadas. As demais distribuições percentuais de publicações foram de $14 \%$ no ano de $2013,25 \%$ no ano de $2015,20 \%$ no ano de 2015 e finalmente $12 \%$ no ano de 2017.

Os 58 artigos científicos encontrados, foram publicados em 34 periódicos diferentes. Tais artigos se encontram nas áreas de: medicina, fisioterapia, enfermagem e psicologia. Dentre as revistas, a área de medicina recebeu maior porcentagem, sendo que 16 tinham enfoque na neurologia, 2 revistas eram específicas sobre EM e outras 2 sobre doença autoimune de forma geral. Nenhuma revista brasileira foi identificada que se dedique apenas para EM, mas duas revistas brasileiras apareceram na lista com artigos sobre o tema: Arquivos de Neuro-Psiquiatria e Fisioterapia em Movimento (Caneda, Vecino, 2016; Figueiredo, Polachini, \& Prado, 2016). Arquivos de Neuro-Psiquiatria é publicado pela Academia Brasileira de Neurologia e a revista Fisioterapia em Movimento pela Pontifícia Universidade Católica do Paraná. Entre os artigos 57 são prospectivos e 2 deles são retrospectivos com revisão de registros clínicos e pesquisa de banco de dados (Tabela 1). 
Tabela 1

Distribuição dos artigos científicos por periódico

\begin{tabular}{|c|c|c|}
\hline Revista / Periódico & $\mathbf{N}$ & $\%$ \\
\hline Multiple Sclerosis Journal & 8 & 13,79 \\
\hline Journal of Neurology & 4 & 6,90 \\
\hline Neurology & 4 & 6,90 \\
\hline Arquivos de Neuro-Psiquiatria & 3 & 5,17 \\
\hline Journal of the Neurological Sciences & 3 & 5,17 \\
\hline Multiple Sclerosis and Related Disorders & 3 & 5,17 \\
\hline Plos One & 3 & 5,17 \\
\hline Archives of Physical Medicine and Rehabilitation & 2 & 3,45 \\
\hline BioMed Central Neurology & 2 & 3,45 \\
\hline Clinical Neurophysiology & 2 & 3,45 \\
\hline Restorative Neurology Neuroscience. & 2 & 3,45 \\
\hline Acta Neurologica Scandinavica & 1 & 1,72 \\
\hline BMC Research Notes & 1 & 1,72 \\
\hline Brain Imaging and Behavior & 1 & 1,72 \\
\hline Clinical Rehabilitation & 1 & 1,72 \\
\hline European Neurology & 1 & 1,72 \\
\hline Fisioterapia em Movimento & 1 & 1,72 \\
\hline International Journal of Molecular Sciences & 1 & 1,72 \\
\hline International Journal of Psychology & 1 & 1,72 \\
\hline Iranian Journal of Allergy, Asthma and Immunology & 1 & 1,72 \\
\hline Journal of Clinical and Experimental Neuropsychology & 1 & 1,72 \\
\hline $\begin{array}{l}\text { Journal of neuroengineering and rehabilitation BioMed Central } \\
\text { Ltd }\end{array}$ & 1 & 1,72 \\
\hline Journal of Neuroscience Nursing & 1 & 1,72 \\
\hline Journal of Neuroimmunology & 1 & 1,72 \\
\hline Lancet Neurology & 1 & 1,72 \\
\hline Magnetic Resonance Imaging & 1 & 1,72 \\
\hline Neural Plasticity & 1 & 1,72 \\
\hline Neurocase: The Neural Basis of Cognition & 1 & 1,72 \\
\hline Neurodegenerative disease management & 1 & 1,72 \\
\hline Neurorehabilitation and Neural Repair & 1 & 1,72 \\
\hline Psychopharmacology (Berl) & 1 & 1,72 \\
\hline Radiology & 1 & 1,72 \\
\hline Rehabilitation Psychology & 1 & 1,72 \\
\hline Total & 58 & 100 \\
\hline
\end{tabular}

Nota. Fonte: Dados coletados para revisão sistemática.

Após a análise do conjunto de 58 artigos selecionados, observou-se que a somatória total nos artigos pesquisados foi de 4194 pessoas clinicamente diagnosticado 
com EM, cujas idades variam entre 12 até 78 anos, com média de 44,30 anos de idade. Sobre o tempo de evolução da doença foi calculada uma média de tempo de 11,41 anos, com o máximo de tempo de 20 anos e no mínimo 7 meses de diagnóstico. A média de tempo de escolaridade obtida nos artigos foi de 13,71 anos, todavia, 23 estudos não citaram o nível de estudo da sua amostra, mesmo sendo uma variável influenciável na avaliação cognitiva (Tabela 2).

\section{Tabela 2}

Dados Demográficos das Amostras dos Estudos Incluídos

\begin{tabular}{lc}
\hline Dados Demográficos & Total \\
\hline Média da Idade (anos) & 44,30 \\
Média da Educação (anos) & 13,71 \\
Duração média da doença (anos) & 11,41 \\
\hline Tipos Clínicos de EM* & Total \\
\hline EMRR & $51(86 \%)$ \\
EMSP & $30(50 \%)$ \\
EMPP & $20(33 \%)$ \\
\hline Média EDSS & 3,38 \\
\hline Nota. EMRR = Esclerose Múltipla Remitente Recorrente; EMSP = Esclerose Múltipla Secundária. EMPP = Esclerose \\
Múltipla Primária Progressiva; EDSS = Escala Expandida do Estado de Incapacidade. As variáveis como idade, \\
educação e tempo de diagnóstico são apresentadas com média e desvio padrão. *Os tipos clínicos de EM foram obtidos \\
ao expressar a proporção de cada tipo com o total de artigos. O percentual de estudos supera os 100\% pois alguns \\
estudos apresentaram mais de um tipo clínico da EM.
\end{tabular}

Entre artigos que usaram metodologia experimental ou quase-experimental, 46 artigos utilizam controles, em que grupos de pacientes foram comparados ao aplicar ou não as intervenções propostas. Os experimentos foram realizados com pessoas com EM para observar padrões cognitivos. Houve também 2 artigos que fizeram estudos comparativos de pessoas com diagnóstico de EM e grupos de participantes com Neuromielite óptica e Comprometimento Cognitivo Leve.

A Tabela 3 permite visualizar cada artigo classificado, com subtipo clínico da EM e a Escala Expandida do Estado de Incapacidade de Kurtzke (EDSS) dos participantes (alguns artigos não mostraram o número da EDSS da amostra avaliada e na célula da tabela colocou-se "NI"-não informado). De forma geral percebeu-se que em relação ao subtipo clínico, 23 artigos enfocaram apenas na EMRR, 10 avaliaram 2 subtipos clínicos; e 17 avaliaram os três subtipos clínicos na pesquisa. 
A EDSS é utilizada para quantificar o grau de incapacidades ocorridas durante a evolução da EM (Kurtzke, 1983). A EDSS foi utilizada em 44 estudos, compondo a média total dos artigos que utilizaram a escala de 3,36 pontos $(\mathrm{DP}=1,4)$. Os resultados evidenciaram valor mínimo de EDSS de 1 ponto mostrando que a amostra desta pesquisa estava sem incapacidades (Ehling et al., 2015) até o máximo de 5,8 pontos no EDSS (Ford-Johnson et al., 2016) que representa pacientes que deambulavam até 200 metros sem ajuda ou descanso e tinham limitações nas atividades diárias. $\mathrm{O}$ estudo com maior pontuação de EDSS apresentou uma amostra composta por participantes mais idosos $(\mathrm{M}=60,78 ; \mathrm{DP}=4,71)$ com diagnóstico de EMSP. Sendo que os escores mais altos do EDSS serão de maior disfunção do quadro. O estudo de Pereira (2013), expõe uma correlação direta entre o grau de incapacidade medido pelo EDSS e o comprometimento das funções cognitivas.

Tabela 3

Descrição dos estudos selecionados que realizaram avaliação neuropsicológica na sua metodologia.

\begin{tabular}{|c|c|c|}
\hline Referências & Subtipo Clínico & EDSS média \\
\hline Bilgi et al. (2015). & EMRR, EMSP & 5,58 \\
\hline Briken et al. (2014). & EMPP, EMSP & 4,95 \\
\hline Cambron et al. (2014). & EMPP, EMSP & NI \\
\hline Campbell et al. (2016). & EMRR, EMSP & 4,43 \\
\hline Caneda et al. (2016). & EMRR & 3,4 \\
\hline Carr et al. (2014). & EMRR, EMSP, EMPP & NI \\
\hline Cerasa et al. (2013). & EMRR & 2,5 \\
\hline Chan et al. (2017). & EMSP & 5,8 \\
\hline Charvet et al. (2017). & EMPP, EMRR, EMSP & 3,5 \\
\hline Chiaravalloti et al.(2013). & EMRR, EMPP, EMSP & NI \\
\hline Covey et al. (2017). & EMRR e EMSP & 2,79 \\
\hline De Giglio et al. (2016). & EMRR & 2 \\
\hline Dehning et al. (2014). & EMRR & 2,87 \\
\hline Devonshire et al. (2016). & EMRR, EMSP & 1,8 \\
\hline Ehling R. et al. (2015). & EMRR & 1 \\
\hline Eijlers et al. (2017). & EMPP, EMRR, EMSP & 3 \\
\hline Ernst et al. (2015). & EMRR & 2,63 \\
\hline Figueiredo et al. (2016). & EMRR & NI \\
\hline Ford-Johnson et al. (2016). & EMPP, EMRR, EMSP & 7,9 \\
\hline Gich et al. (2015). & EMRR, EMSP & 2,7 \\
\hline Hanssen et al. (2016). & EMRR, EMPP, EMSP & 4,3 \\
\hline Henneghan et al. (2017). & EM & NI \\
\hline Hoang et al. (2016). & EMRR, EMPP, EMSP & 4,15 \\
\hline Hughes et al (2015). & EMPP, EMRR & 4,1 \\
\hline Janssen et al.(2015). & EMRR & 2,77 \\
\hline Jensen et al. (2016). & EMPP, EMRR, EMSP & 5,6 \\
\hline Kappos L. et al. (2016). & EMRR & NI \\
\hline
\end{tabular}


Tabela 3

Descrição dos estudos selecionados que realizaram avaliação neuropsicológica na sua metodologia (continuação)

\begin{tabular}{|c|c|c|}
\hline Referências & Subtipo Clínico & EDSS média \\
\hline Koenig et al. (2014). & EMRR e EMSP & 1,5 \\
\hline Kuusisto, et al. (2016). & $\begin{array}{c}\text { EMRR, } \\
\text { EMSP, EMPP }\end{array}$ & 3,9 \\
\hline Leavitt et al. (2014). & EMRR, EMPP, EMSP & NI \\
\hline Maarouf et al. (2017). & EMRR & 1,5 \\
\hline Maghzi et al. (2014). & EM & NI \\
\hline Magnin et al. (2015). & EMPP, EMRR, EMSP & 5,3 \\
\hline Mäntynen et al. (2014). & EMRR & NI \\
\hline $\begin{array}{l}\text { Martínez-González e Piqueras. } \\
\text { (2014). }\end{array}$ & EMRR & NI \\
\hline Mattioli et al. (2016). & EMRR & 2,5 \\
\hline Mäurer et al. (2013). & EMRR, SPMS & $\mathrm{NI}$ \\
\hline Mokhber et al. (2014). & EM & 2,24 \\
\hline Morrow et al. (2013). & EMRR, EMSP & 3,55 \\
\hline Morrow e Rosehart. (2015). & EMPP, EMRR, EMSP & 3,5 \\
\hline Morrow et al. (2017). & EMPP,EMRR, EMSP & 3 \\
\hline Müller, et al. (2013). & EMSP & NI \\
\hline Palm et al. (2016). & EMPP, EMRR, EMSP & 4,2 \\
\hline Parada Fernández, et al. (2015). & EMPP, EMRR, EMSP & NI \\
\hline Patti et al. (2013). & EMRR & NI \\
\hline Pavsic et al. (2015). & EMPP, EMRR, EMSP & 6 \\
\hline Pedullà et al. (2016). & EMRR, EMSP & 3,8 \\
\hline Peyro et al. (2016). & EMRR & 3,2 \\
\hline Roostaei et al. (2015). & EMRR & 1,28 \\
\hline Sandroff et al. (2014). & EMRR & NI \\
\hline Sandroff et al. (2016). & EMRR & 3 \\
\hline Settle et al (2015). & EMPP, EMRR, EMSP & NI \\
\hline Sundgren et al. (2015). & EMRR & 2,7 \\
\hline Svenningsson et al. (2013). & EM & 3,2 \\
\hline Vanotti et al. (2013). & EMRR & 2,2 \\
\hline Vanotti et al. (2014). & EMRR & 1,3 \\
\hline Zecca et al. (2014). & EMRR & 3 \\
\hline Zhang et al. (2016). & EMRR & 3,38 \\
\hline
\end{tabular}

Nota: EM = Esclerose Múltipla; EMRR = Esclerose Múltipla Remitente Recorrente; EMSP = Esclerose Múltipla Secundária Progressiva; EMPP = Esclerose Múltipla Primária Progressiva; EDSS = Escala Expandida do Estado de Incapacidade; NI= não informado.

As três baterias de avaliação mais frequentes utilizadas nos artigos de forma completa foram: Brief International Cognitive Assessment of Multiple Sclerosis (BICAMS), Multiple Sclerosis Functional Composite Measure (MSFC) e Brief Repeatable Battery of Neuropsychological Test (BRB-N). A BICAMS abrange os testes cognitivos Symbol Digit Modalities Test, California Verbal Learning Test e Brief Visuospatial Memory Test, foi utilizado em cinco artigos. O MSFC compõe-se dos instrumentos Timed 25-Foot Walk, 9-Hole Peg Test e Paced Auditory Serial Addition Test (empregado em 7 artigos) O BRB-N é o mais abrangente e é composto por Selective 
Reminding Test, Spacial Recall Test, Symbol Digit Modalities Test, Paced Auditory Serial Addition Test, Word List Generation. O BRB-N foi empregado de forma completa por 11 publicações e outras 12 publicações utilizaram apenas alguns subtestes.

Os testes específicos utilizados com mais frequência foram: Paced Auditory Serial Addition Test (PASAT), Symbol Digit Modalities Test (SDMT), Trail Making Test (TMT) e Wechsler Adult Intelligence Scale (WAIS). Em todos os artigos que o WAIS foi utilizado, foram selecionados subtestes específicos e não a escala geral, o subteste mais empregado foi Dígitos na ordem direta e inversa. A Escala Wechsler Abreviada de Inteligência (WASI) foi usada duas vezes. A Tabela 4 também apresenta a frequência em que os instrumentos foram utilizados nos estudos investigados.

Tabela 4

Instrumentos e baterias neuropsicológicos selecionados após triagem

\begin{tabular}{|c|c|c|c|}
\hline Instrumentos & $\mathrm{N}$ de estudos & $\%$ & Função \\
\hline Paced Auditory Serial Addition Test & 30 & 50,8 & $\begin{array}{c}\text { Velocidade de } \\
\text { processamento e }\end{array}$ \\
\hline Symbol Digit Modalities Test & 23 & 39,0 & $\begin{array}{c}\text { Memória Operacional } \\
\text { Velocidade de } \\
\text { Processamento }\end{array}$ \\
\hline Trail Making Test & 11 & 18,6 & Flexibilidade Cognitiva \\
\hline Wechsler Adult Intelligence Scale & 11 & 18,6 & Capacidade intelectual \\
\hline Verbal Fluency Test & 10 & 16,9 & $\begin{array}{c}\text { Fluência verbal } \\
\text { semântica e fonética }\end{array}$ \\
\hline Controlled Oral Word Association Test & 5 & 8,5 & Fluência verbal \\
\hline Stroop Test & 5 & 8,5 & Controle Inibitório \\
\hline Rey Auditory-Verbal Learning Test & 5 & 8,5 & Memória Verbal \\
\hline Wisconsin Card Sorting Test & 3 & 5,1 & Flexibilidade Cognitiva \\
\hline Wechsler Memory Scale & 3 & 5,1 & Memória \\
\hline Escala Wechsler Abreviada de Inteligência (WASI) & 2 & 3,4 & Capacidade Intelectual \\
\hline
\end{tabular}

Com exceção de uma pesquisa (Settle et al., 2015), que optou por avaliação cognitiva a distância por meio do instrumento Automated Neuropsychological Assessment Metrics, as demais fizeram avaliação presencial. Entre os estudos, apenas um estudo, que não realizou intervenção, utilizou delineamento longitudinal, com acompanhamento ao longo de três anos por meio de testes cognitivos e comparações com ressonância magnética (Maghzi et al., 2014). 


\section{Discussão}

O presente estudo teve como foco listar os instrumentos de avaliação neuropsicológica empregados em artigos publicados no período de 2013 até 2017. A pesquisa evidenciou uma grande variabilidade nos testes e suas formas de administração, o que corrobora os dados da revisão sistemática publicados no período de 1997 a 2009 (Ferreira, 2010). Esta revisão relatou uma ausência da uniformidade na bateria de avaliação cognitiva em EM e sugeriu que a avaliação neuropsicológica da EM ainda estava em processo de construção e consolidação.

As três baterias específicas que se destacaram para avaliar pessoas com diagnóstico de EM foram BICAMS, MSFC e BRB-N. Tais baterias são breves, facilmente aplicadas e a literatura sugere que seriam importantes na detecção precoce de casos que precisam de investigação completa. Percebeu-se nas pesquisas que os testes que fazem parte das baterias são tanto utilizados em conjunto como separados. BICAMS e BRB-N se limitam a avaliação cognitiva. A BICAMS abrange três testes que avaliam diferentes funções (memória visual, memória verbal e velocidade de processamento), enquanto o BRB-R avalia a velocidade de processamento na modalidade visual, memória de trabalho na alça fonológica, memória verbal, memória viso-espacial e fluência verbal espontânea. Já a bateria MSFC analisa uma única função cognitiva que é a memória de trabalho, mas acrescenta a avaliação das funções motoras dos membros superiores e inferiores. A escolha das baterias dependeu dos objetivos da pesquisa e da necessidade de uma avaliação motora em conjunto.

O teste mais utilizado foi PASAT, que pertence às baterias MSFC e BRB-N para avaliação dos pacientes com diagnóstico de EM, e foi utilizado em conjunto com baterias padronizadas ou separadamente. O teste SDMT, foi o segundo mais utilizado na amostra da pesquisa, e foi o único teste que faz parte de todas as baterias cognitivas que são recomendadas especificamente para pessoas com EM, incluindo BRB-N e BICAMS já citados. O teste foi utilizado em conjunto com as baterias em 10 artigos e acrescentado com outros testes neuropsicológicos por 12 artigos (20,3\%). Foi também empregado em um artigo como único teste cognitivo na avaliação. 
O PASAT e o SDMT avaliam entre outras funções cognitivas a velocidade de processamento da informação em pessoas com EM (Rao, Leo, \& Aubin-Farbert, 1989). Tal análise, com enfoque na velocidade de processamento foi possível observar em todos os artigos dessa revisão que utilizaram os testes supracitados. O prejuízo na velocidade de processamento é uma disfunção cognitiva frequentemente observada em pessoas com EM, que resulta em impacto significativo na qualidade de vida e foi considerada a melhor medida para discriminar pessoas com EM de controles saudáveis. Nas publicações da amostra a velocidade de processamento esteve associada a prejuízos secundários em outras funções cognitivas como memória e aprendizagem (Manca, Sharrack, Paling, Wilkinson, \& Venneri, 2018).

Percebeu-se nas pesquisas que houve uma permanência e frequência similar no decorrer do tempo em relação aos dois testes. Sendo que esses testes foram utilizados tanto separadamente como na mesma testagem. Um possível fato da grande utilização do PASAT e SDMT é que eles fazem parte da maioria das baterias mais utilizadas para avaliação cognitiva de EM. Entretanto, estudos tem apontado o PASAT como um teste muito difícil e de alta resistência para a população geral que tem nível de educação formal limitado ao ensino fundamental, uma vez que demanda raciocínio lógico matemático e conhecimentos de aritmética. Outro aspecto importante que pode colaborar para que o PASAT tenha um grau de dificuldade maior para a população geral seria o fato dele aliciar recursos da memória de trabalho também. Possivelmente ele não seja o ideal para triagem, avaliação e seguimento da cognição em paciente com EM (Brooks et al., 2011; Gow \& Deary, 2004). Já em um estudo francês, sobre a detecção precoce de distúrbios cognitivos, mostrou que o SDMT apresenta sensibilidade de $74,2 \%$, especificidade de $76,9 \%$ e a acurácia de 75,4\%. O que faz o SDMT colaborar na seleção de pacientes para bateria cognitiva mais completas (Deloire et al., 2006).

Outro teste utilizado com frequência foi Trail Making Test que fornece também dados sobre a velocidade de processamento, mas seu enfoque nas pesquisas foi a função executiva de flexibilidade cognitiva. Em todos os artigos o TMT foi utilizado em conjunto com no mínimo mais cinco testes ou em protocolos maiores como de 15 testes cognitivos, mostrando que seu enfoque não foi de rastreio cognitivo, mas de aprofundamento e com baterias completas. $\mathrm{O}$ estudo das funções executivas em pessoas com EM é relativamente 
recente, sendo que as primeiras pesquisas que abordam essas funções foram publicadas na década de 1980.

Pesquisa realizada por Cerezo, Martín e Aladro (2015), teve como principal objetivo analisar o perfil de alteração das funções executivas em uma amostra de 100 pessoas com EM. Entre outros testes (Verbal Fluency Test, Stroop Test Teste, Wisconsin Card Sorting Test e WAIS), utilizou o Trail Making Test e concluiu que os componentes mais prejudicados foram: flexibilidade cognitiva, inibição e capacidade de abstração, mostrando evidências de prejuízos das funções executivas em pessoas com EM, principalmente em estágios mais avançados. A pesquisa também descreveu que os pacientes com pior performance foram aqueles com formas clínicas progressivas e altos índices no EDSS.

Os dados normativos do teste PASAT e SDMT, para amostra brasileira, foram encontrados em conjunto com os estudos da escala MSFC e BICAMS, respectivamente, e as pesquisas utilizaram grupo controle e grupo de pessoas com EM (Haase, Lima, Lacerda, \& Lana-Peixoto, 2004; TilberyI et al., 2005; Spedo, 2016). A padronização do TMT não foi encontrada com amostra de pessoas com EM, entretanto, em relação a adultos saudáveis foi realizada uma padronização por Zimmermann, Cardoso, Kristensen e Paz Fonseca (2017), em que foram investigados os efeitos da idade e escolaridade no desempenho da tarefa.

Em relação aos testes de memória (Rey Auditory-Verbal Learning Test e Escala de Memória de Weschsler), percebeu-se que os mais utilizados são testes referendados na literatura. Entretanto, apenas quatro artigos utilizaram instrumentos para avaliação de memória. Todavia, a pesquisa de Rosti-Otajärvi e Hämäläinen (2014) mostra que aproximadamente $40 \%$ a $60 \%$ das pessoas com EM têm problemas de memória, sendo uma das funções mais afetadas.

Por fim, os estudos revisados mostraram disfunções cognitivas nos pacientes diagnosticados com EM. Os principais prejuízos cognitivos específicos citados nos artigos foram: memória verbal, memória de trabalho, atenção, fluência verbal e funções executivas (controle inibitório e velocidade de processamento). Vários artigos referiram que pacientes com EM obtiveram escores menores em diversos testes neuropsicológicos se comparados com o grupo controle saudável. Tais deficiências cognitivas podem 
interferir na participação de atividades sociais e laborais. Além disso, observa-se a necessidade de acompanhar esse público de forma longitudinal para capturar aspectos da progressão da doença.

O presente estudo verificou que houve variabilidade metodológica dos estudos, diversidade dos instrumentos selecionados para avaliar as funções cognitivas na EM e observou-se ainda que os instrumentos neuropsicológicos utilizados nos estudos da amostra precisariam de maior detalhamento.

\section{Conclusão}

A revisão sistemática apresentada mostrou a importância da investigação das funções cognitivas em pacientes com EM. Todos os artigos pesquisados evidenciaram que pacientes com diagnóstico de EM estão associados com algum grau de disfunção cognitiva. As funções cognitivas específicas afetadas não mostram consenso ao serem citadas nos artigos, possivelmente devido a variações metodológicas das avaliações e a variabilidade das alterações anatômicas que ocorrem na EM.

As baterias breves mais utilizadas evidenciadas na revisão sistemática são BICAMS, MSFC e BNR-N. Já os testes mais utilizados na avaliação neuropsicológica de paciente com EM são PASAT e SDMT, tendo enfoque na velocidade de processamento. Em suma, o levantamento realizado no presente estudo indicou que uma bateria de testes neuropsicológicos para avaliar as funções cognitivas de pessoas com EM deve investigar minimamente as funções de atenção, velocidade de processamento da informação, memória verbal e funções executivas (flexibilidade cognitiva, memória de trabalho e controle inibitório). No Brasil, alguns dos testes utilizados nos estudos da amostra são adaptados e padronizados e, portanto, podem ser incluídos nesta bateria: WAIS, Fluência Verbal, Trail Making Test, Rey Auditory Verbal Learning Test, Wisconsin Card Sorting Test. 


\section{Referências}

Benedict, R. H. B., Weinstock-Guttman, B., Fishman, I., Sharma, J., Tjoa, C. W., \& Bakshi, R. (2004). Prediction of Neuropsychological Impairment in Multiple Sclerosis: Comparison of Conventional Magnetic Resonance Imaging Measures of Atrophy and Lesion Burden. Archives of Neurology, 61(2), 226-230. Doi.org/10.1001/archneur.61.2.226

Brooks, J. B. B., Giraud, V. O., Saleh, Y. J., Rodrigues, S. J., Daia, L. A., \& Fragoso, Y. D. (2011). Paced auditory serial addition test (PASAT): a very difficult test even for individuals with high intellectual capability. Arquivos de Neuro-Psiquiatria, 69(3), 482-484. Doi.org/10.1590/S0004-282X2011000400014

Caneda, M. A., \& Vecino, M. C. A. D. (2016). The correlation between EDSS and cognitive impairment in MS patients. Assessment of a Brazilian population using a BICAMS version. Arquivos de neuro-psiquiatria, 74(12), 974-981. Doi.org/10.1590/0004-282x20160151

Deloire, M. S., Bonnet, M. C., Salort, E., Arimone, Y., Boudineau, M., Petry, K. G., \& Brochet, B. (2006). How to detect cognitive dysfunction at early stages of multiple sclerosis? Multiple Sclerosis Journal, 12(4), 445-452. Doi.org/10.1191/1352458506ms1289oa

Ehling, R., Di Pauli, F., Lackner, P., Rainer, C., Kraus, V., Hegen, H., ... Berger, T. (2015). Impact of glatiramer acetate on paraclinical markers of neuroprotection in multiple sclerosis: A prospective observational clinical trial. Journal of Neuroimmunology, 287, 98-105. https://doi.org/10.1016/j.jneuroim.2015.08.004

Ferreira, M. L. B. (2010). Cognitive deficits in multiple sclerosis: a systematic review. Arquivos de Neuro-psiquiatria, 68(4), 632-641. Doi.org/10.1590/S0004282X2010000400029

Figueiredo, A. I., Polachini, C. R. N., \& Prado, A. L. C. (2016). Assessment of patients with multiple sclerosis according to tests of the Multiple Sclerosis Functional Composite. Fisioterapia em Movimento, 29(4), 677-684. Doi.org/10.1590/19805918.029.004.ao03

PsicolArgum. 2019jan./mar., 37(95), 125-142. 
Ford-Johnson, L., DeLuca, J., Zhang, J., Elovic, E., Lengenfelder, J., \& Chiaravalloti, N. D. (2016). Cognitive effects of modafinil in patients with multiple sclerosis: A clinical trial. Rehabilitation Psychology, 61(1), 82-91.Doi.org/10.1037/a0039919

Gow, A. J., \& Deary, I. J. (2004). Is the PASAT past it? Testing attention and concentration without numbers. Journal of Clinical and Experimental Neuropsychology, 26(6), 723-736. Doi.org/10.1080/13803390490509295

Haase, V. G., Lima, E. D. P., Lacerda, S. S., \& Lana-Peixoto, M. A. (2004). Desenvolvimento da versão brasileira da multiple sclerosis functional composite measure (MSFC-BCTRIMS). Arquivos de Neuropsiquiatria, 62(2-A), 363-70. Recuperado de http://www.scielo.br/pdf/\%0D/anp/v62n2a/a33v622a.pdf

Jüni, P., \& Egger, M. (2009). PRISMAtic reporting of systematic reviews and metaanalyses. The Lancet, 374(9697), 1221-1223. Doi.org/10.1016/S01406736(09)61765-7

Kurtzke, J. F. (1983). Rating neurologic impairment in multiple sclerosis: An expanded disability status scale (EDSS). Neurology, 33(11), 1444-1452. Doi.org/10.1212/wnl.33.11.1444

Lanz, M., Hahn, H. K., \& Hildebrandt, H. (2007). Brain atrophy and cognitive impairment in multiple sclerosis: a review. Journal of Neurology, 254(2), 43-48. Doi.org/10.1007/s00415-007-2011-8

Leavitt, V. M., Tosto, G., \& Riley, C. S. (2018). Cognitive phenotypes in multiple sclerosis. Journal of Neurology, 265(3), 562-566. Doi.org/10.1007/s00415-018-87475

Maghzi, A. H., Revirajan, N., Julian, L. J., Spain, R., Mowry, E. M., Liu, S. \& Waubant, E. (2014). Magnetic resonance imaging correlates of clinical outcomes in early multiple sclerosis. Multiple Sclerosis and Related Disorders, 3(6), 720-727. Doi.org/10.1016/j.msard.2014.07.003

Manca, R., Sharrack, B., Paling, D., Wilkinson, I. D., \& Venneri, A. (2018, May 15). Brain connectivity and cognitive processing speed in multiple sclerosis: A systematic review. Journal of the Neurological Sciences, 388, pp. 115-127. Doi.org/10.1016/j.jns.2018.03.003 
Özakbaş, S. (2015). Cognitive impairment in multiple sclerosis: Historical aspects, current status, and beyond. Noropsikiyatri Arsivi, 52, pp. S12-S15. Doi.org/10.5152/npa.2015.12610

Patti, F., Nicoletti, A., Messina, S., Bruno, E., Fermo, S. Lo, Quattrocchi, G., ... Zappia, M. (2015). Prevalence and incidence of cognitive impairment in multiple sclerosis: a population-based survey in Catania, Sicily. Journal of Neurology, 262(4), 923-930. Doi.org/10.1007/s00415-015-7661-3

Pereira, A. G. (2013). Evolução das funções cognitivas psíquicas e motoras dos pacientes portadores de esclerose múltipla. (Tese de doutorado, Programa de Pós- Graduação em Medicina e Ciências da Saúde, Pontifícia Universidade Católica do Rio Grande do Sul. Porto Alegre). Recuperado de http://repositorio.pucrs.br/dspace/handle/10923/4420

Rao, S. M., St. Aubin-Faubert, P., \& Leo, G. J. (1989). Information processing speed in patients with multiple sclerosis. Journal of Clinical and Experimental Neuropsychology, 11(4), 471-477. Doi.org/10.1080/01688638908400907

Rinaldi, F., Calabrese, M., Grossi, P., Puthenparampil, M., Perini, P., \& Gallo, P. (2010). Cortical lesions and cognitive impairment in multiple sclerosis. Neurological Sciences, 31(2), 235-237. Doi.org/10.1007/s10072-010-0368-4

Rosti-Otajärvi, E. M., \& Hämäläinen, P. I. (2014). Neuropsychological rehabilitation for multiple sclerosis. Cochrane Database of Systematic Reviews, 2. Doi.org/10.1002/14651858.CD009131.pub3

Tilbery, C. P.; Mendes, M. F.; Thomaz, R. B.; de Oliveira, B. E. S.; Kelian, G. L. R.; Busch, R.; ... Paula CaleffiI. (2005). Padronização da multiple sclerosis functional composite measure (MSFC) na população brasileira. Arquivos de Neuro-Psiquiatria, 63 (01), 127-132. Recuperado de http://www.scielo.br/pdf/\%0D/anp/v63n1/23611.pdf Zimmermann, N., Cardoso, C. de O., Kristensen, C. H., \& Fonseca, R. P. (2017). Normas brasileiras e efeitos de idade e escolaridade no Teste Hayling e no Teste de Trilhas. Trends in Psychiatry and Psychotherapy, 39(3), 188-195. Doi.org/10.1590/22376089-2016-0082 